\title{
Solving the Crisis in Big-Bang Nucleosynthesis by the Radiative Decay of an Exotic Particle
}

\author{
Erich Holtmann ${ }^{a, b}$, Masahiro Kawasaki ${ }^{c}$ and Takeo Moroi ${ }^{b}$ \\ ${ }^{a}$ Department of Physics, University of California, Berkeley, CA 94720 \\ ${ }^{b}$ Theoretical Physics Group, Lawrence Berkeley Laboratory, University of California, Berkeley, \\ CA 94720 \\ ${ }^{c}$ Institute for Cosmic Ray Research, University of Tokyo, Tokyo 188, JAPAN
}

(September 17, 2018)

\begin{abstract}
We discuss a new mechanism which can solve the crisis in standard bigbang nucleosynthesis. A long-lived particle $X\left(10^{4}\right.$ sec $\lesssim \tau_{X} \lesssim 10^{6}$ sec $)$ which decays into photon(s) will induce cascade photons, and destroy significant amounts of $\mathrm{D}$ and ${ }^{3} \mathrm{He}$ without destroying ${ }^{4} \mathrm{He}$ or too much ${ }^{7} \mathrm{Li}$. We numerically investigate this process and derive a constraint on the properties of $X$ such that the theoretical values of the primordial light-element abundances agree with observation. We also present some candidates for the unstable particle $X$.
\end{abstract}


The abundances of the light elements are a crucial test of the big-bang nucleosynthesis (BBN) scenario, since BBN precisely predicts the primordial (i.e., before stellar processing) abundances of $\mathrm{D},{ }^{3} \mathrm{He},{ }^{4} \mathrm{He}$, and ${ }^{7} \mathrm{Li}[\mathrm{I}]$. In the past, improvements in the theoretical and observational estimates of the light elements seemed to give us a better confirmation of the standard BBN scenario.

Recently, however, observations seem to conflict with the theoretical predictions of standard BBN: if we determine the baryon-to-photon ratio $\eta$ by using the observed ${ }^{4} \mathrm{He}$ abundance, then the primordial abundances of $\mathrm{D}$ and ${ }^{3} \mathrm{He}$ predicted from the standard $\mathrm{BBN}$ calculation are too large. In particular, based on a statistical argument, Hata et al. claimed that the standard BBN scenario is inconsistent with observations at a very high confidence level [2]. Furthermore, Copi et al. pointed out that the systematic error in the ${ }^{4} \mathrm{He}$ observation has likely been underestimated if the standard BBN scenario is correct. These arguments suggest the need for some modification of the theoretical or observational estimations of the primordial abundances.

At present, there are several attempts to solve the discrepancy, though none of them has been confirmed. One way is to adopt some modification of the observed abundance. In particular, if we assume an extra systematic error in the estimation of the ${ }^{4} \mathrm{He}$ abundance or if we trust the higher measurements of $\mathrm{D}$ in the primordial $\mathrm{H}_{\mathrm{I}}$ cloud, then we will have better agreement between theory and observation [3]. Alternatively, the models of chemical and stellar evolution of the light elements may need some change. The other way to solve the problem is to modify the standard scenario of BBN. For example, one can reduce the ${ }^{4} \mathrm{He}$ abundance by allowing degenerate electron neutrinos [4], or by assuming a massive unstable neutrino with mass about $1 \mathrm{MeV}$ and lifetime about $1 \mathrm{sec}$ [ [5. Since BBN, together with the Hubble expansion of the universe and the cosmic microwave background radiation (CMBR), is one of the most essential predictions of the big-bang cosmology, it is important to clarify the origin of the discrepancy.

In this letter, we propose another new mechanism to solve the crisis by reducing the predicted D and ${ }^{3} \mathrm{He}$ abundance. Essentially, if some exotic particle $X$ decays into photons 
well after BBN, then the predictions of standard BBN can be modified. Conventionally, such radiatively decaying particles have been regarded as sources of cosmological difficulties [6-9]. However, we can actually have a better scenario of BBN if the cascade photons can destroy significant amounts of $\mathrm{D}$ and ${ }^{3} \mathrm{He}$ without affecting ${ }^{4} \mathrm{He}$ and ${ }^{7} \mathrm{Li}$. Therefore, in this letter, we derive a constraint on the properties of $X$ which makes the BBN scenario viable. We also suggest several candidates for the unstable particle $X$ based on realistic particle physics models.

First of all, we briefly review the effects of the radiative decay of $X$. Once a high energy photon is emitted into the thermal bath, cascade processes are induced. The high energy photon, electron, and positron spectra are formed accordingly. Cascade photons then induce photodissociation of the light elements. Thus, if the radiative decay of $X$ induces many cascade photons at a time $t$ after BBN starts $(t \gtrsim 1 \mathrm{sec})$, then the predictions of standard BBN can be modified. In the photon cascade process, double photon pair creation $\left(\gamma+\gamma_{\mathrm{BG}} \rightarrow e^{+}+e^{-}\right.$, with $\gamma_{\mathrm{BG}}$ being the photon in thermal background) plays an important role: almost all the photons with energy larger than $\epsilon_{\mathrm{thr}} \sim m_{e}^{2} / 22 T$ scatter off photons in the thermal background and become $e^{+} e^{-}$pairs, since the typical event rate for this process is much larger than that of other processes. On the other hand, for photons with energy smaller than the threshold energy $\epsilon_{\mathrm{thr}} \sim m_{e}^{2} / 22 T$, this process is extremely suppressed by kinematics [10,8,9]. As a result, radiative decay of $X$ may cause photodissociation of light elements only if the decay of $X$ occurs at $T \lesssim m_{e}^{2} / 22 Q$, with $Q$ being the threshold energy of photodissociation. For example, photodissociation of ${ }^{4} \mathrm{He}$, whose binding energy is about $20 \mathrm{MeV}$, can be effective only for $T \lesssim 500 \mathrm{eV}$, i.e. for $t \gtrsim O\left(10^{6-7} \mathrm{sec}\right)$.

The effects of the radiative decay of $X$ essentially depend on the following parameters: the lifetime $\tau_{X}$ of $X$, the number density of $X$, and the energy $\epsilon_{\gamma 0}$ of the photons emitted in $X$ decay. In this letter, for simplicity, we assume that $X \rightarrow \gamma+\gamma$ with a $100 \%$ branching ratio, so $\epsilon_{\gamma 0}=m_{X} / 2$. (For the effect of the hadronic decay of $X$, see Ref. [11].) To parameterize the abundance of $X$, we use the yield variable $Y_{X}$, which is the ratio of the number density of $X$ to that of photons: $Y_{X}=n_{X} / n_{\gamma}$. The yield variable is essentially the number of $X$ in 
a comoving volume, and it evolves with time as $Y_{X}=Y_{X 0} e^{-t / \tau_{X}}$. Therefore, once $\tau_{X}, Y_{X 0}$, $\epsilon_{\gamma 0}$, and $\eta$ are fixed, we can calculate the primordial abundances of the light elements.

The procedure used in this letter is as follows. We first solve the Boltzmann equations for the distribution functions of the high energy photons and electrons in order to determine the abundance of cascade photons. Then, combining the derived cascade spectrum with the modified Kawano code [12] in which effects of the photodissociation are taken into account, we calculate the primordial abundances of the light elements, $y_{2 \mathrm{p}}, y_{3 \mathrm{p}}$, and $Y_{\mathrm{p}}$, where $y_{2 \mathrm{p}}$ and $y_{3 \mathrm{p}}$ are the primordial number fraction of $\mathrm{D}$ and ${ }^{3} \mathrm{He}$ relative to hydrogen $\mathrm{H}$, respectively, and $Y_{\mathrm{p}}$ is the primordial mass fraction of ${ }^{4} \mathrm{He}$. (For details, see Refs. [8,9].)

These theoretical predictions should be compared with the constraints obtained from observations. First, let us discuss the constraints on D and ${ }^{3} \mathrm{He}$. Since D is only destroyed after BBN, the mass fraction of D decreases with time, and hence [13]

$$
\begin{aligned}
y_{2, \text { ism }} & \leq y_{2 \mathrm{p}} R_{X}, \\
y_{2 \odot} & \leq y_{2 \mathrm{p}} R_{X},
\end{aligned}
$$

where $R_{X}=X_{\mathrm{H}, \mathrm{p}} / X_{\mathrm{H}, \text { now }}, y_{2}$ is the number fraction of $\mathrm{D}$ relative to $\mathrm{H}, X_{\mathrm{H}}$ is the mass fraction of $\mathrm{H}$, and the subscripts "ism", "๑", and "now" denote the abundances in interstellar matter, in the solar system, and in the present universe, respectively. Furthermore, taking into account the chemical evolution of $\mathrm{D}$ and ${ }^{3} \mathrm{He}$, we obtain a third constraint [13]:

$$
\begin{array}{r}
\left\{-y_{2 \mathrm{p}}+\left(\frac{1}{g_{3}}-1\right) y_{3 \mathrm{p}}\right\} y_{2 \odot}-\frac{1}{g_{3}} y_{2 \mathrm{p}} y_{3 \odot} \\
+\left(y_{2 \mathrm{p}}^{2}+y_{2 \mathrm{p}} y_{3 \mathrm{p}}\right) R_{X} \leq 0
\end{array}
$$

where $y_{3}$ is the number fraction of ${ }^{3} \mathrm{He}$ relative to $\mathrm{H}$, and $g_{3}$ is the survival fraction of ${ }^{3} \mathrm{He}$ in stellar processes. Usually, $g_{3}$ is estimated to be $0.25-0.5$ [14].

From observation, the present abundances of the light elements (with 1- $\sigma$ error) are known to be [15]

$$
y_{2, \text { ism }}=(1.6 \pm 0.2) \times 10^{-5} \text {, }
$$




$$
\begin{aligned}
& y_{2 \odot}=(2.57 \pm 0.92) \times 10^{-5}, \\
& y_{3 \odot}=(1.52 \pm 0.34) \times 10^{-5}, \\
& R_{X}=1.1 \pm 0.04 .
\end{aligned}
$$

Here, we briefly comment on the detection of $\mathrm{D}$ in the primordial $\mathrm{H}_{\mathrm{I}}$ cloud. The absorbed line observed in Ref. [16] suggests $y_{2} \simeq 2.5 \times 10^{-4}$, which is much larger than the value given in (田) and (5). However, it is claimed that the observed absorption line may be due to a Doppler-shifted hydrogen line [17]. Therefore, in this paper, we do not adopt the D abundance obtained in Ref. [16].

For fixed values of $y_{2 \mathrm{p}}, y_{3 \mathrm{p}}$, and $g_{3}$, by regarding $y_{2, \text { ism }}, y_{2 \odot}, y_{3 \odot}$, and $R_{X}$ as statistical variables which obey Gaussian distributions, we can calculate the probability $P\left(y_{2 \mathrm{p}}, y_{3 \mathrm{p}} ; g_{3}\right)$ that the conditions (11) - (3) are satisfied simultaneously:

$$
\begin{array}{r}
P\left(y_{2 \mathrm{p}}, y_{3 \mathrm{p}} ; g_{3}\right)=\int_{\mathbf{V}} d y_{2, \text { ism }} d y_{2 \odot} d y_{3 \odot} d R_{X} \\
\times f\left(y_{2, \text { ism }} ; \bar{y}_{2, \text { ism }}, \sigma_{y_{2, \text { ism }}}^{2}\right) f\left(y_{2 \odot} ; \bar{y}_{2 \odot}, \sigma_{y_{2 \odot}}^{2}\right) \\
\quad \times f\left(y_{3 \odot} ; \bar{y}_{3 \odot}, \sigma_{y_{3 \odot}}^{2}\right) f\left(R_{X} ; \bar{R}_{X}, \sigma_{R_{X}}^{2}\right) .
\end{array}
$$

In Eq.(8), the integration is performed in the volume $\mathbf{V}$ in which all the constraints (迎) (3) are satisfied, and $f\left(x ; \bar{x}, \sigma^{2}\right)$ is the Gaussian distribution function for $x$ with mean $\bar{x}$ and variance $\sigma^{2}$.

By using the probability $P\left(y_{2 \mathrm{p}}, y_{3 \mathrm{p}} ; g_{3}\right)$, we can obtain a constraint on the primordial abundance of $\mathrm{D}$ and ${ }^{3} \mathrm{He}$. For a fixed value of $g_{3}$, we exclude the parameter set $\left(y_{2 \mathrm{p}}, y_{3 \mathrm{p}}\right)$ if $P\left(y_{2 \mathrm{p}}, y_{3 \mathrm{p}} ; g_{3}\right)<0.05$. Here, we note that our $P\left(y_{2 \mathrm{p}}, y_{3 \mathrm{p}} ; g_{3}\right)=0.05$ contour with $g_{3}=0.25$ is almost the same as the $95 \%$ C.L. constraint of Hata et al. in Ref. [13. That is, comparing the constraints on $y_{3 \mathrm{p}}$ for a fixed value of $y_{2 \mathrm{p}}$, we checked that the discrepancy between the two approaches is at most $(10-20) \%$; hence, we conclude that they are consistent.

Next, we quote the constraint on the primordial abundance of ${ }^{4} \mathrm{He}$, as estimated from the low-metallicity $\mathrm{H}_{\mathrm{II}}$ regions [18]:

$$
Y_{\mathrm{p}}=0.232 \pm 0.003(\text { stat }) \pm 0.005 \text { (syst) }
$$


We are now ready to show our numerical results. In our analysis, we compare the constraint on the baryon-to-photon ratio $\eta$ derived from (1) - (3), with the contours which yield the correct primordial abundance of ${ }^{4} \mathrm{He}$. In Fig. 1, we show the contour for the $5 \%$ probability constraints in the $m_{X} Y_{X 0}$ vs. $\eta$ plane for several values of $\tau_{X}$. Here, we take the mass of $X$ to be $1 \mathrm{TeV}$, a neutron lifetime of 887.0sec [19], and $g_{3}=0.25$. Also, we show the contours which will yield specified abundances of ${ }^{4} \mathrm{He}$.

The typical behavior of the constraint can be understood as follows. If $\epsilon_{\gamma 0} Y_{X}$ is too small, then the abundance of cascade photons is so suppressed that the standard BBN scenario is almost unchanged. In this case, constraints from $\mathrm{D}$ and ${ }^{3}$ He prefer $\eta$ to be $(3-7) \times 10^{-10}$, which is too large to be consistent with the ${ }^{4} \mathrm{He}$ constraint. However, if $\epsilon_{\gamma 0} Y_{X}$ has the correct value, then sufficient amounts of $\mathrm{D}$ and ${ }^{3} \mathrm{He}$ are destroyed by cascade photons, and a smaller value of $\eta$ is allowed. (Remember that, in the standard BBN scenario, smaller values of $\eta$ give larger values of $y_{2 \mathrm{p}}$ and $y_{3 \mathrm{p}}$.) In particular, as can be seen in Fig. 1, $Y_{\mathrm{p}}=0.232$ may be consistent with the constraints from $\mathrm{D}$ and ${ }^{3} \mathrm{He}$ for $g_{3}=0.25$. Furthermore, we also checked that, even for $g_{3}=0.5, Y_{\mathrm{p}}=0.235$ is acceptable if $\epsilon_{\gamma 0} \sim 10 \mathrm{MeV}$. Thus, if there is an exotic particle, we can have a better agreement between observation and the theoretical prediction. If $\epsilon_{\gamma 0} Y_{X 0}$ is too large, then too much $\mathrm{D}$ and ${ }^{3} \mathrm{He}$ are destroyed; hence such a parameter region is excluded. Notice that the distribution function of cascade photons depends primarily on the total amount of injected energy [9] if the mass of $X$ is much larger than $\epsilon_{\mathrm{thr}}$. In this case, our results are almost independent of $m_{X}$ for fixed $m_{X} Y_{X 0}$.

One may worry that too much ${ }^{7} \mathrm{Li}$ may be photodissociated in this scenario, since the threshold for the photodissociation of ${ }^{7} \mathrm{Li}$ is very low $(\sim 2.5 \mathrm{MeV})$. However, the cross section for the photodissociation of ${ }^{7} \mathrm{Li}$ is about four times smaller than that of $\mathrm{D}$ dissociation, so the abundance of ${ }^{7} \mathrm{Li}$ is not reduced much compared with the $\mathrm{D}$ abundance. Numerically, we checked that $(30-40) \%$ of ${ }^{7} \mathrm{Li}$ is photodissociated in our scenario. For example, for $\eta=2 \times 10^{-10}$, we get $n_{7} \mathrm{Li} / n_{\mathrm{H}}=(0.7-1.0) \times 10^{-10}$, and a larger abundance of ${ }^{7} \mathrm{Li}$ is obtained if we use a larger or smaller value of $\eta$. (Notice that we prefer $\eta \lesssim 3 \times 10^{-10}$. For this range, ${ }^{7} \mathrm{Li}$ production though ${ }^{7} \mathrm{Be}$ is not important. For $\eta \gtrsim 3 \times 10^{-10}$, we cannot predict ${ }^{7} \mathrm{Li}$ 
abundance since the cross section for ${ }^{7}$ Be photofission is not available.) Taking into account the uncertainty in the observed abundance of ${ }^{7} \mathrm{Li}$, viz. $n_{7} \mathrm{Li} / n_{\mathrm{H}}=\left(1.4_{-0.7}^{+2.1}\right) \times 10^{-10}$ [20], the ${ }^{7} \mathrm{Li}$ abundance in our scenario is consistent with observation. Furthermore, in our scenario ${ }^{6} \mathrm{Li}$ can be produced via ${ }^{7} \mathrm{Li}(\gamma, n){ }^{6} \mathrm{Li}$. This reaction may produce much more ${ }^{6} \mathrm{Li}$ than standard BBN does. Numerically, we expect a few $\%$ of ${ }^{7} \mathrm{Li}$ can be converted into ${ }^{6} \mathrm{Li}$ for

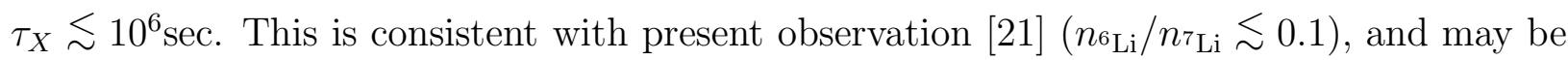
checked in future observations.

Let us now discuss the constraints on $\tau_{X}$. First of all, the blackbody spectrum of CMBR observed by COBE [22] gives us a severe constraint on particles with lifetime longer than $\sim 10^{6} \sec$ [23], which is when the double Compton process $\left(\gamma+e^{-} \rightarrow \gamma+\gamma+e^{-}\right)$freezes out. In particular, if $\epsilon_{\gamma 0} Y_{X 0} \sim 10^{-9} \mathrm{GeV}$, then $\tau_{X} \gtrsim 10^{6} \mathrm{sec}$ is forbidden [23]. Furthermore, if $\tau_{X} \gtrsim 10^{6} \mathrm{sec}$ and $\epsilon_{\gamma 0} \gtrsim 20 \mathrm{GeV}$, then cascade photons destroy ${ }^{4} \mathrm{He}$ effectively, which may result in the overproduction of $\mathrm{D}$ and ${ }^{3} \mathrm{He}$. Thus, we have an upper bound on $\tau_{X}$ of $\sim 10^{6}$ sec. On the other hand, as $\tau_{X}$ decreases, the threshold for double photon pair creation $\epsilon_{\text {thr }}$ becomes low, and the number of photons contributing to $\mathrm{D}$ and ${ }^{3} \mathrm{He}$ photodissociation decreases, for a fixed value of $\epsilon_{\gamma 0} Y_{X 0}$. In this case, a larger value of $m_{X} Y_{X 0}$ is required in order to destroy sufficient amounts of D and ${ }^{3} \mathrm{He}$, as can be seen in Fig. 囵. However, a larger initial mass density of $X$ would speed up the expansion of the universe, so ${ }^{4}$ He may be overproduced. These arguments exclude $\tau_{X}$ shorter than $\sim 10^{4} \mathrm{sec}$. As a result, to make our scenario viable, we must adopt $10^{4} \sec \lesssim \tau_{X} \lesssim 10^{6}$ sec. In Fig. 2, we show the allowed region (i.e., the region where $\left.P\left(y_{2 \mathrm{p}}, y_{3 \mathrm{p}} ; g_{3}\right) \geq 0.05\right)$ on the $\tau_{X}$ vs. $m_{X} Y_{X 0}$ plane. Here, we use $g_{3}=0.25$, and we vary $\eta$ so that $Y_{\mathrm{p}}=0.232$ (dotted contour) or 0.235 (solid contour).

Finally, we suggest several candidates for the unstable particle $X$, specifically within supergravity [24] models. In such models, there may be several particles which have a long lifetime $\left(10^{4} \mathrm{sec} \lesssim \tau_{X} \lesssim 10^{6} \mathrm{sec}\right)$. Probably the most famous such particle is the gravitino, whose cosmological implications have been well-investigated [7,8]. As we will see below, the gravitino may have the properties required to solve the crisis in BBN, although in the past it has been regarded as a source of cosmological difficulties (the so-called gravitino problem). 
We can quantitatively check whether the gravitino can solve the difficulty in BBN, since its interactions are almost unambiguously determined [24]. If the gravitino decays only into a photon and photino pair, then its lifetime is estimated as

$$
\tau_{X} \simeq 4 \times 10^{5} \sec \times\left(1 \mathrm{TeV} / m_{3 / 2}\right)^{3}
$$

where $m_{3 / 2}$ is the gravitino mass. Thus, if the gravitino mass is about $1 \mathrm{TeV}$, its lifetime is about $10^{5-6}$ sec, which is appropriate for our purpose. Notice that the photino produced by the gravitino decay interacts very weakly with the thermal background. Thus, our results can be applied to the gravitino if we rescale the $m_{X} Y_{X 0}$-axis by a factor of $\sim 0.5$. Furthermore, assuming an inflationary universe, the gravitino abundance is determined just after the reheating period, and depends only on the reheat temperature $T_{R}[8]$ :

$$
Y_{X 0} \simeq 2 \times 10^{-11}\left(T_{R} / 10^{10} \mathrm{GeV}\right)
$$

For $m_{3 / 2} \sim 1 \mathrm{TeV}, T_{R} \sim 10^{8-9} \mathrm{GeV}$ gives $\epsilon_{\gamma 0} Y_{X 0} \sim 10^{-(9-10)} \mathrm{GeV}$. We note here that $T_{R} \sim 10^{9} \mathrm{GeV}$ may be realized in the chaotic inflation model [25] if the inflaton field decays though gravitational interactions.

If the gravitino is the lightest superparticle, then we can construct another scenario. In this case, the next-to-the lightest superparticle (NLSP), which we assume to be the photino, decays into a gravitino and a photon. By a loose tuning of the parameters, the NLSP can be identified with an unstable particle $X$ which solves the difficulty in BBN. Thus, in supergravity models, we have several candidates for the unstable particle $X$ which can make the $\mathrm{BBN}$ scenario viable.

In summary, an exotic particle with lifetime $\tau_{X} \sim 10^{4-6}$ sec can solve the crisis in BBN if $m_{X} Y_{X 0}$ is tuned within a factor of 2-3. Candidates for $X$ include the gravitino and the photino, both of which naturally appear in supergravity models. Of course, even in other types of models, one may be able to find a candidate for the radiatively decaying particle $X$.

The authors thank N. Hata and D. Thomas for useful discussions and comments. This 
work was supported in part by DOE under Contract DE-AC03-76SF00098 and in part by NSF under grant PHY-90-21139. 


\section{REFERENCES}

[1] T.P. Walker, G. Steigman, D.N. Schramm, K.A. Olive, and H.-S. Kang, Astrophys. J. 376 (1991) 51.

[2] N. Hata, R.J. Scherrer, G. Steigman, D Thomas, T.P. Walker, S. Bludman, and P. Langacker, Phys. Rev. Lett. 75 (1995) 3977.

[3] C. J. Copi, D. N. Schramm, and M. S. Turner, Phys. Rev. Lett. 75 (1995) 3981.

[4] K.A. Olive, D.N. Schramm, D. Thomas, and T.P. Walker, Phys. Lett. B265 (1991) 239; H.-S. Kang and G. Steigman, Nucl. Phys. B372 (1992) 494.

[5] M. Kawasaki, P. Kernan, H.-S. Kang, R.J. Scherrer, G. Steigman, and T.P. Walker, Nucl. Phys. B419 (1994) 105; S. Dodelson, G. Gyuk, and M. S. Turner, Phys.Rev. D49 (1994) 5068.

[6] D. Lindley, Mon. Not. Roy. Astron. Soc. B188 (1979) 15P; D. Lindley, Astrophys. J. 294 (1985) 1.

[7] H. Pagels and J.R. Primack, Phys. Rev. Lett. 48 (1982) 223; S. Weinberg, Phys. Rev. Lett. 48 (1982) 1303; L.M. Krauss, Nucl. Phys. B227 (1983) 556; J. Ellis, E. Kim, and D.V. Nanopoulos, Phys. Lett. B145 (1984) 181; R. Juszkiewicz, J. Silk, and A. Stebbins, Phys. Lett. B158 (1985) 463; M. Kawasaki and K. Sato, Phys. Lett. B189 (1987) 23; J. Ellis, G.B. Gelmini, J.L. Lopez, D.V. Nanopoulos, and S. Sarkar, Nucl. Phys. B373 (1992) 399; T. Moroi, H. Murayama, and M. Yamaguchi, Phys. Lett. B303 (1993) 289.

[8] M. Kawasaki and T. Moroi, Prog. Theor. Phys. 93 (1995) 879.

[9] M. Kawasaki and T. Moroi, Astrophys. J. 452 (1995) 506.

[10] A.A. Zdziarski, Astrophys. J. 335 (1988) 786; R. Svensson and A.A. Zdziarski, Astrophys. J. 349 (1990) 415. 
[11] M.H. Reno and D. Seckel, Phys. Rev. D37 (1988) 3441; S. Dimopoulos, R. Esmailzadeh, L.J. Hall, and G.D. Starkman, Astrophys. J. 330 (1988) 545; Nucl. Phys. B311 (1989) 699.

[12] L. Kawano, preprint FERMILAB-Pub-92-04-A.

[13] G. Steigman and M. Tosi, Astrophys. J. 453 (1995) 173; N. Hata, R.J. Scherrer, G. Steigman, D. Thomas, and T.P. Walker, Astrophys. J. 458 (1996) 637.

[14] D.S.P. Dearborn, D.N. Schramm, and G. Steigman, Astrophys. J. 302 (1986) 35; G. Steigman and M. Tosi, Astrophys. J. 401 (1992) 150.

[15] P.R. McCullough, Astrophys. J. 390 (1992) 213; J.L. Linsky et al., Astrophys. J. 402 (1993) 694; J. Geiss, in Origin and Evolution of the Elements, edited by N. Prantzos, E. Vangioni-Flam, and M. Casse (Cambridge University Press, Cambridge, 1993) p89; G. Steigman and M. Tosi in Ref. [14].

[16] A. Sangalia, L.L. Cowie, C.J. Hogan, and M. Rugers, Nature 368 (1994) 599.

[17] G. Steigman, preprint OSU-TA-22-94 (astro-ph/9410060).

[18] K.A. Olive and G. Steigman, Astrophys. J. Suppl. 97 (1995) 49.

[19] Particle Data Group, Phys. Rev. D50 (1994) 1173.

[20] C. J. Copi, D. N. Schramm, and M. S. Turner, Science 267 (1995) 192.

[21] V.V. Smith, D.L. Lambert, and P.E. Nissen; Astrophys. J. 408 (1993) 262, L.M. Hobbs and J.A. Thorburn, Astrophys. J. 428 (1994) L25.

[22] J.C. Mather et al., Astrophys. J 420 (1994) 439.

[23] W. Hu and J. Silk, Phys. Rev. Lett. 70 (1993) 2661.

[24] E. Cremmer, S. Ferrara, L. Girardello, and A. van Proeyen, Nucl. Phys. B212 (1983) 413. 
[25] A.D. Linde, Phys. Lett. B129 (1983) 177. 


\section{FIGURES}

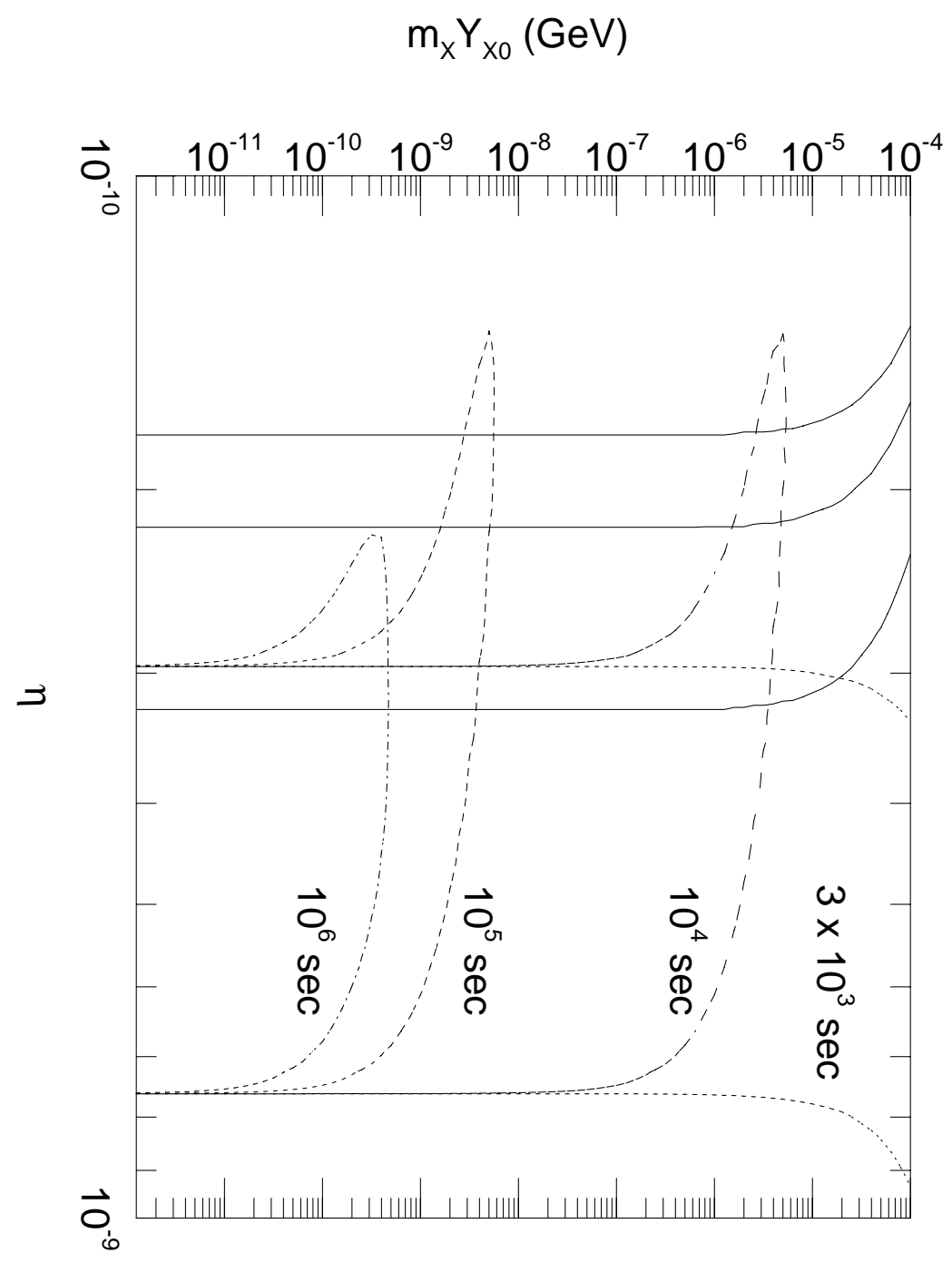

FIG. 1. Constraints from ${ }^{3} \mathrm{He}$ and D, $P\left(y_{2 \mathrm{p}}, y_{3 \mathrm{p}} ; g_{3}\right) \geq 0.05$, shown in the $\eta$ vs. $m_{X} Y_{X 0}$ plane for $m_{X}=1 \mathrm{TeV}$, and $g_{3}=0.25$. The lifetime of $X$ is take to be $3 \times 10^{3} \mathrm{sec}$ (dotted line), $10^{4} \mathrm{sec}$ (long-dashed line), $10^{5} \mathrm{sec}$ (short-dashed line), and $10^{6} \mathrm{sec}$ (dotted-dashed line). The allowed regions are the interiors of the curves. The solid lines are the contours which yield $Y_{\mathrm{p}}=0.232,0.235$, and 0.240 (from left to right) for $\tau_{X}=3 \times 10^{3}$ sec. 


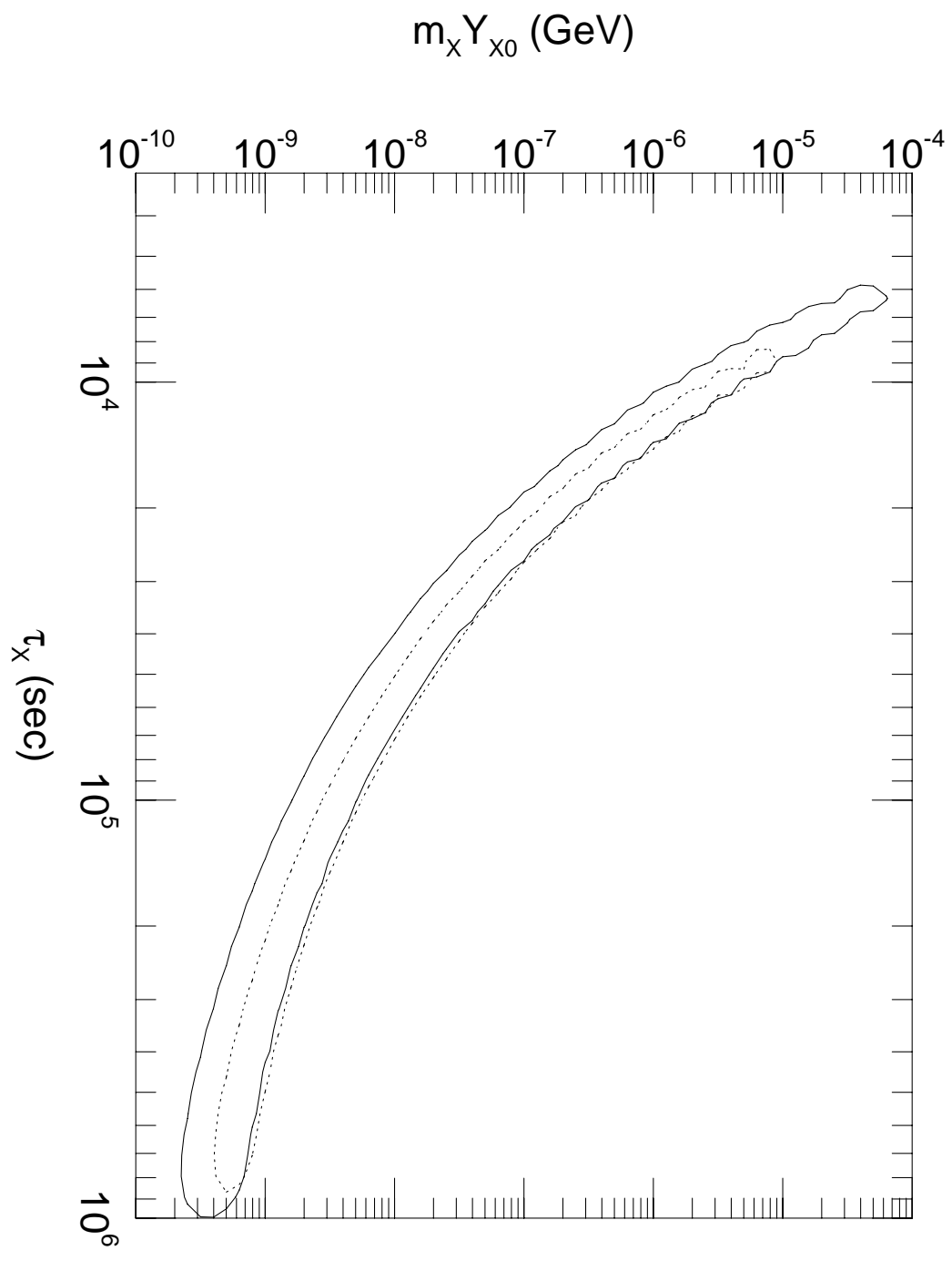

FIG. 2. The contour for $P\left(y_{2 \mathrm{p}}, y_{3 \mathrm{p}} ; g_{3}\right)=0.05$ with $m_{X}=1 \mathrm{TeV}$ and $g_{3}=0.25 . \eta$ is determined so that $Y_{\mathrm{p}}=0.232$ (dotted) and 0.235 (solid). Notice that the interiors of the contours are favored. 